\title{
Treatment of walled-off pancreatic necrosis: when and how?
}

\section{(ㄷ)(1) $\Theta$}

\author{
Author \\ Enrique Vazquez-Sequeiros \\ Institution \\ Endoscopy Unit, Gastroenterology Division, University \\ Hospital Ramón y Cajal, IRYCIS, Madrid, Spain \\ Bibliography \\ DOI https://doi.org/10.1055/s-0043-116380 | \\ Endoscopy International Open 2017; 05: E1060-E1061 \\ (c) Georg Thieme Verlag KG Stuttgart · New York \\ ISSN 2364-3722
}

\author{
Corresponding author \\ Enrique Vazquez-Sequeiros MD, PhD, Endoscopy Unit, \\ Gastroenterology Division, University Hospital Ramón y \\ Cajal, IRYCIS Madrid, Carretera de Colmenar km 9,100, \\ 28034 Madrid, Spain \\ Fax: +34918835348 \\ evazquezse@gmail.com
}

Endoscopic drainage of pancreatic fluid collections (PFC) is a minimally invasive technique that has mostly replaced surgical therapies in this type of patients [1 -4]. Endosonography (EUS)guided PFC drainage is preferred by most clinicians over external radiological drainage due to better efficacy and lower adverse events $(A E)$ rate [4-7]. However, a number of questions regarding EUS-guided drainage of PFC are yet to be answered, such as when to perform drainage or which technique is better suited to resolve these difficult cases. The existing evidence for these questions is still limited and mostly based on uncontrolled and non-comparative data.

In the current issue of the journal, the Seattle group reports interesting data that may have a significant impact in clinical practice [8]. Results from the Sahar study provide some evidence against using modern and costly lumen-apposing metal stents (LAMS) to drain PFC [8]. Sahar et al conducted a study comparing outcomes of 2 different EUS-guided techniques: double pigtail plastic stent (DPS) vs lumen-apposing metal stents (LAMS), associated with percutaneous drainage for treatment of walled-off pancreatic necrosis (WON). Twentyfive patients were treated in each group and results showed similar technical success ( $100 \%$ in each group), hospitalization days (14.5 vs 13.1), time to resolution of WON (77 vs 63 days) and AEs ( 24 vs $32 \%$ ). Therefore, these results may lead us to conclude that LAMS do not appear to improve DPS results when combined with percutaneous drainage and are associated with higher costs, and thus their use should be discouraged. These results and conclusions may modify the growing shift in clinical practice towards using metal stents instead of plastic for PFC drainage. In fact, this type of practice is based on no comparative data demonstrating to date that metal stents are more efficient.
However, these results should be cautiously analyzed before jumping into definitive conclusions that may not be entirely true. First of all, it has to be acknowledged that this is a retrospective comparison of 2 treatment groups with the same sample size, and apparently comparable baseline characteristics. It is well known that selection and recall bias are likely to occur in retrospective analysis and that may unfortunately limit the validity of these interesting and provocative results. Furthermore, as noted by the authors, the study has a reduced sample size that may certainly increase the likelihood of a false-negative result. If we assume that, for example, a $10 \%$ difference between techniques (DPS vs LAMS) is clinically meaningful, approximately 80 to 90 patients would need to be included in each treatment group to have statistical power $(80 \%-90 \%)$ adequate to detect differences if they really exist. Therefore, we cannot be sure if the negative results are due to inadequate statistical power. To definitively answer that question, a larger prospective study with adequate statistical power is still required.

Despite these methodological issues, there are also several technical factors that deserve further discussion. Drainage of PFC may be technically challenging, especially if you are not working at a tertiary referral center, and your case volume is not elevated. Readers should be aware that authors performing EUS-guided PFC drainage in this study are not "average" endoscopists, but real experts in this type of therapy, with a large experience with PFC drainage and with the double drainage technique. Technical success with EUS-guided PFC drainage has been shown to be below the $100 \%$ reported in the Sahar study when a study includes institutions that do not have significant expertise $[8,9]$. The technical mistakes in such cases are associated with access to the PFC with the cystotome or the stent, or with loss of guidewire access during exchange of accessories [9]. It has been hypothesized that technical mistakes, more 
prone to happen in the hands of less experienced endoscopists, may be minimized by reducing the number of steps required for PFC drainage, as occurs with the Hot Axios LAMS (cystotome and balloon dilation steps are avoided). However, although it seems reasonable, this hypothesis is yet to be proven.

Another point that deserves further discussion is combining percutaneous and endoscopic treatment to treat PFC patients. That type of therapy is not the standard at most institutions. The combined approach is more aggressive therapy and usually reserved for patients who have not responded to endoscopic or percutaneous drainage alone. Therefore, as previously mentioned, this may limit the validity of results to those institutions that routinely perform combined drainage. Furthermore, one may even question if LAMS can be used as a single therapy, as opposed to combined internal and external percutaneous drainage. If both therapies were equally effective, one may also argue that by using LAMS for PFC drainage, patient quality of life would improve significantly, and the cost of percutaneous drainage would be saved.

Because most institutions use a single drainage technique for PFC collections, what is really needed in clinical practice are prospective and controlled studies comparing DPS vs LAMS vs straight biliary fully covered self-expandable metal stents (SBFCSEMS). Those studies should separately analyze pancreatic pseudocysts and WON. Existing evidence from the literature suggests that EUS-guided drainage of PFC with placement of DPS is effective in pseudocysts, but not for WON, probably due to debris and necrosis resulting in stent occlusion and treatment failure $[10,11]$. Large retrospective studies have shown that LAMS and SBFCSEMS appear to be more effective than DPS (90\%, $95 \%$ and $81 \%$, respectively, $P=0.001)$ as a single therapy in WON $[9,12-16]$. Although not specifically designed to compare LAMS vs SBFCSEMS, initial reports have not shown differences in efficacy between different types of metal stents, but have suggested that early AEs may be more frequent in patients treated with LAMS $[9,16]$.

\section{Conclusion}

In summary, the interesting and controversial data regarding PFC drainage are mainly useful for institutions that employ a double drainage technique. In those cases, it does appear that LAMS may not offer any advantage over DPS. However, if a single drainage technique is the approach at your institution, we are still waiting for strong evidence to support use of DPS or LAMS. Until that moment, LAMS or SBFCSEMS appears to be better suited than DPS to treat complicated patients with WON.

\section{Competing interests}

$$
\text { None }
$$

\section{References}

[1] Banks PA, Bollen TL, Dervenis C et al. Classification of acute pancreatitis-2012: revision of the Atlanta classification and definitions by international consensus. Gut 2013; 62: 102-111

[2] Yasuda I, Nakashima M, Iwai T et al. Japanese multicenter experience of endoscopic necrosectomy for infected walled-off pancreatic necrosis: The JENIPaN study. Endoscopy 2013; 45: 627-634

[3] Holt BA, Varadarajulu S. The endoscopic management of pancreatic pseudocysts (with videos). Gastrointest Endosc 2015; 81: 804-812

[4] Varadarajulu S, Bang JY, Sutton BS et al. Equal efficacy of endoscopic and surgical cystogastrostomy for pancreatic pseudocyst drainage in a randomized trial. Gastroenterology 2013; 145: 583-590

[5] Yusuf TE, Baron TH. Endoscopic transmural drainage of pancreatic pseudocysts: results of a national and an international survey of ASGE members. Gastrointest Endosc 2006; 63: 223 - 227

[6] Kwon YM, Gerdes H, Schattner MA et al. Management of peripancreatic fluid collections following partial pancreatectomy: a comparison of percutaneous versus EUS-guided drainage. Surg Endosc 2013; 27: $2422-2427$

[7] Keane MG, Sze SF, Cieplik N et al. Endoscopic versus percutaneous drainage of symptomatic pancreatic fluid collections: a 14-year experience from a tertiary hepatobiliary centre. Surg Endosc 2015: [Epub ahead of print]. doi:10.1007/s00464-015-4668-x

[8] Sahar N, Kozarek R, Kanji Z et al. Do lumen apposing metal stents (LAMS) improve treatment outcomes of walled-off pancreatic necrosis over plastic stents using dual modality drainage? Endosc Internat Open 2017; 05: 1052 - 1059

[9] Vazquez-Sequeiros E, Baron TH, Perez-Miranda M et al. Evaluation of short and long-term efficacy effectiveness and safety of fully covered self-expandable metal stents for drainage of pancreatic fluid collections: results of a Spanish nationwide registry. Gastrointest Endosc 2016; 84: 450 - 457

[10] Bang DY, Varadarajulu S. Metal versus plastic stent for transmural drainage of pancreatic fluid collections. Clin Endosc 2013; 46: 500 502

[11] Baron TH, Thaggard WG, Morgan DE et al. Endoscopic therapy for organized pancreatic necrosis. Gastroenterology 1996; 111: 755-764

[12] Shah R, Shah JN, Waxman I et al. Safety and efficacy of endoscopic ultrasound-guided drainage of pancreatic fluid collections with lumen-apposing covered self-expanding metal stents. Clinical Gastroenterology and Hepatology 2015; 13: 747-752

[13] Sharaiha RZ, DeFilippis EM, Kedia P et al. Metal versus plastic for pancreatic pseudocyst drainage: clinical outcomes and success. Gastrointest Endosc 2015; 82: 822-827

[14] Rinniella E, Kunda R, Dollhopf M et al. EUS-guided drainage of pancreatic fluid collections using a novel lumen-apposing metal stent on an electrocautery-enhanced delivery system: a large retrospective study (with video). Gastrointest Endosc 2015; 82: 1039-1046

[15] Walter D, Will U, Sanchez-Yague A et al. A novel lumen-apposing metal stent for endoscopic ultrasound-guided drainage of pancreatic fluid collections: a prospective cohort study. Endoscopy 2015; 47: $63-67$

[16] Siddiqui AA, Kowalski TE, Loren DE et al. Fully covered self-expanding metal stents versus lumen-apposing fully covered self-expanding metal stent versus plastic stents for endoscopic drainage of pancreatic walled-off necrosis: clinical outcomes and success. Gastrointest Endosc 2017; 85: 758-765 